\title{
Effect of Surfaces of FeNbCuBSiP Ribbons
}

\author{
P. Butvin ${ }^{a, *}$, B. Butvinováa ${ }^{a}$, M. Kuzminski ${ }^{b}$, A. Ślahiska-Waniewska ${ }^{b}$, J. Sitek $^{c}$, \\ I. MAŤKO ${ }^{a}$, M. KADLEČÍKOVÁ ${ }^{d}$ \\ ${ }^{a}$ Institute of Physics, Slovak Academy of Sciences, Dúbravská cesta 9, 84511 Bratislava, Slovakia \\ ${ }^{b}$ Institute of Physics, Polish Academy of Sciences, Al. Lotników 32/46, 02-668 Warszawa, Poland \\ ${ }^{c}$ Institute of Nuclear and Physical Engineering, STU, Ilkovičova 3, 81219 Bratislava, Slovakia \\ ${ }^{d}$ Institute of Electronics and Photonics FEI, STU, Ilkovičova 3, 81219 Bratislava, Slovakia
}

Boron was partially substituted by phosphorus (3 at.\%) in two Si-poor Fe-Nb-Cu-B-Si Finemets. Mostly nonsignificant changes were observed after vacuum annealing at $500{ }^{\circ} \mathrm{C}$, whereas equivalent $\mathrm{Ar}$ annealing resulted in significantly better soft-magnetic properties, which suffer from compressive surfaces stress. Possibly by hampering surface crystallization, the substitution eased the surfaces stress and reduced undesired off-axis anisotrophy at the cost of limiting the useful annealing temperature.

DOI: 10.12693/APhysPolA.126.152

PACS: $75.30 . \mathrm{Gw}, 75.50 . \mathrm{Tt}, 75.60 . \mathrm{Ch}$

\section{Introduction}

Si-poor Finemets show good soft-magnetic properties with significantly higher saturation than "classic" Si-rich compositions. The good properties deteriorate during non-vacuum annealing due to stress exerted by surfaces (crystallizing first) on the ribbon interior. Thus we tried to influence the surfaces by substituting a part of boron by phosphorus in a composition between Si-poor and practically $\mathrm{Nb}, \mathrm{Cu}$ and Si-free one [1].

\section{Materials and methods}

Metallic glass precursor ribbons of Fe78 or $74-\mathrm{Nb3}-$ Cu1-B10-P3-Si5 or 9 at.\% were prepared by the planarflow casting on air. Strips of $10 \mathrm{~mm}$ width and $20-23 \mu \mathrm{m}$ thickness were annealed in vacuum or in Ar ambience at $500{ }^{\circ} \mathrm{C}$ for $1 \mathrm{~h}$. Sample contamination (fingerprints etc.) has been carefully avoided, but no specific surface cleaning was attempted. Hysteresis loops were recorded using a digitizing hysteresisgraph at $21 \mathrm{~Hz}$ sinusoidal $H$ excitation in Helmholtz drive coils, along the ribbon long axis. Magnetic domains were observed by Kerr-effect method (MOKE). Structure, critical temperatures $\left(T_{x}, T_{C}\right)$ and magnetostriction were studied previously [2] and are augmented by Mössbauer spectroscopy (MS) in this work. Electron Dispersive Spectroscopy (EDS) was used to look over differences of element composition between surfaces and bulk. Investigation of surface chemistry of the strips was performed by Raman spectroscopy (RS) using He-Ne laser $(632.8 \mathrm{~nm})$.

\section{Results and discussion}

If compared to non-substituted composition, vacuumannealed Si5 shows a significantly reduced coercivity by $60 \%$ (attaining $6.7 \mathrm{~A} / \mathrm{m}$ ) but also a slightly lower saturation $(1.38 \mathrm{~T})$. The substitution did not improve the coercivity in Si9 but increased saturation to $1.36 \mathrm{~T}$. This

\footnotetext{
*corresponding author; e-mail: pavol.butvin@savba.sk
}

is due to increased Curie temperature - the explanation of this somewhat surprising change will be sought in near future. Otherwise, the upright loop shape after vacuum annealing does not reflect the substitution, whose effect is far more pronounced after Ar annealing, indicating a change of the surface properties. Whereas Si5 continues to show reduced transverse anisotropy after Ar-annealing at even higher temperature, $500^{\circ} \mathrm{C}$ is the upper limit for Si9 to show properties improved by the substitution. Figure 1 shows that annealing at higher temperature, by even $20^{\circ} \mathrm{C}$, causes 4 -fold increase of magnetization work to $41 \mathrm{~J} / \mathrm{m}^{3}$ and $25 \%$ increase of coercivity (from 16 to $20 \mathrm{~A} / \mathrm{m})$.

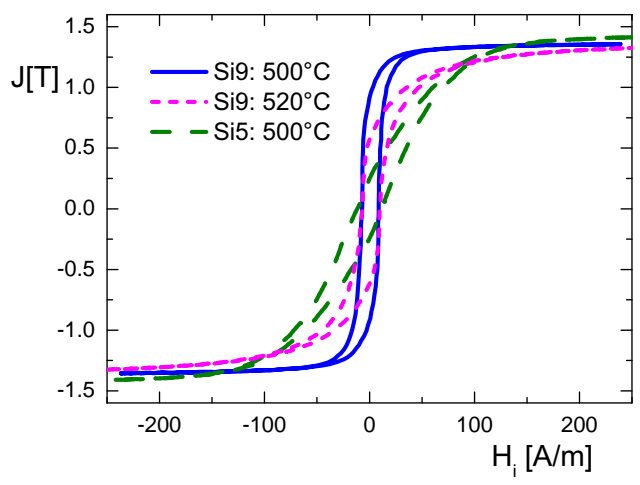

Fig. 1. $21 \mathrm{~Hz}$ loops of Ar-annealed strips.

Since loops corresponding to Fig. 2a are quite upright, they imply that the transversal domains are the minor ones. Since the domains are not magnetized "head-on", they are not the closure domains but stripe domains [3] (of a primary surface structure) and are not considered to fill the ribbon interior. Otherwise, Fig. 2a represents an indication of transversal macroscopic heterogeneity $(\mathrm{TMH})$, which is typical for wider Finemet rib- 
bons [4]. Whereas longitudinal surface anisotropy prevails at the edges, the central part of the surface is governed by transverse anisotropy due to prevailing tension along ribbon axis and across the ribbon axis respectively. The heterogeneous anisotropy comes from magnetoelastic interaction in Si9 because this silicon percentage in Finemets does not support a significant creep-inducedlike anisotropy [5]. The opposite surface (wheel side) displays complementary anisotropy (not shown for Si9, but similar to Fig. 2c), which points to mutually compensated stress on the surfaces. Fig. $2 \mathrm{~b}$ shows the surface anisotropy and thus TMH starting to decline by deceasing transverse stress during surface crystallization (see Fig. 3 - MS).

Vacuum-annealed Si5 also shows TMH, however with principally similar domain structure on both surfaces. This property could be accounted for by assuming that surfaces stress does not compete with each other, but both surfaces compete and establish a balance with the ribbon interior. The advancing surface crystallization in Ar again appears to reduce TMH but unlike Si9, it does not preserve the majority of longitudinal anisotropy. The slant loop of Si5 (Fig. 1) indicates a transverse anisotropy component, as if the two opposite surfaces compress the ribbon interior in a similar manner. Surface removal by etching lets the loop to show significantly reduced tilt and magnetization work (i.e. weaker transverse anisotropy).

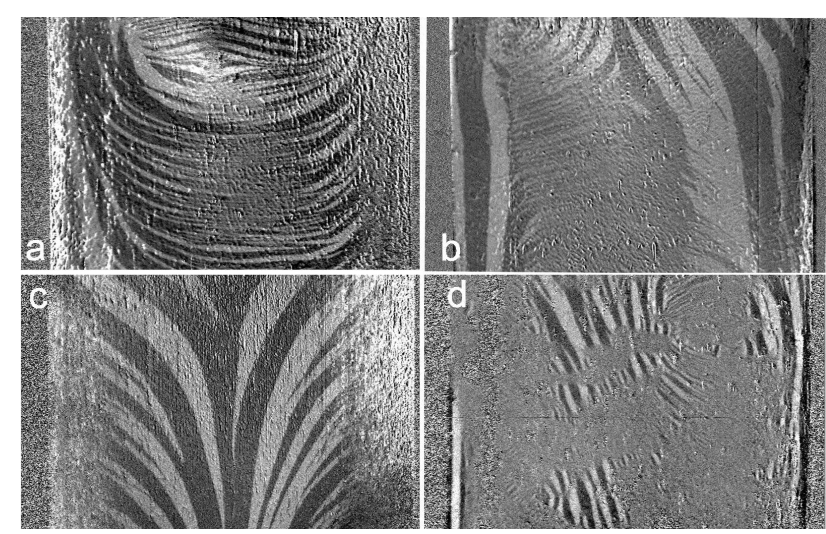

Fig. 2. Domain structures of strips annealed at $500{ }^{\circ} \mathrm{C}$ : (a) Si9 in vacuum, (b) Si9 in $\mathrm{Ar}$, (c) $\mathrm{Si} 5$ in vacuum and (d) Si5 in Ar. Whole ribbon width is seen (demagnetized state). Wheel side is shown on (c), air side otherwise; horizontal magneto-optical sensitivity is used for (a), vertical one otherwise.

EDS proved that there is no appreciable difference in $P$ percentage between surfaces and interior. It confirmed still extensive oxidation of Ar-annealed surfaces. Oxides do not press, but appear to induce - promote surface crystallization (Fig. 3). The oxides identified by RS are mostly iron oxides $\left(\mathrm{Fe}_{2} \mathrm{O}_{3}, \mathrm{Fe}_{3} \mathrm{O}_{4}\right)$. Clear identification of a $\mathrm{P}$ compound was not successful, either. Whereas vacuum annealing of Si9 did not result in well resolved MS subspectra (too weak signal from crystalline-phase
Zeeman splitting), Ar annealing lets partly resolved DO3 superstructure (the four minor subspectra occupying $20 \%$ of spectrum area in Fig. 3) to start forming. There is not enough Si to form perfect DO3. The still Si-poorer Si5 shows larger crystalline share of solid solution of $\mathrm{Si}$ in Fe for Ar-annealing ( $40 \%$ for Ar, $25 \%$ for vacuum). The difference between vacuum and Ar annealing points to surface crystallization.

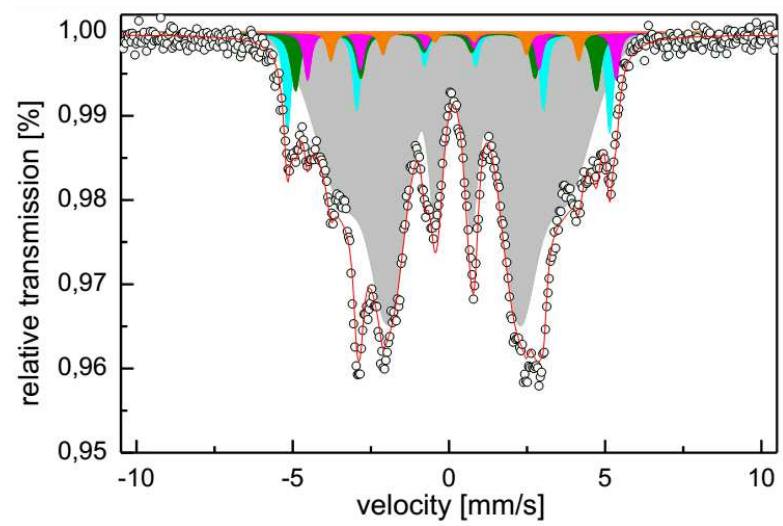

Fig. 3. Mössbauer spectra of Si9 annealed in Ar at $500{ }^{\circ} \mathrm{C}$. The largest-area subspectrum (light gray) belongs to the amorphous rest.

\section{Conclusions}

Although the substitution of 3 at.\% of B by P affects the intrinsic material parameters just slightly and only minor differences are observed after vacuum annealing at $500{ }^{\circ} \mathrm{C}$, it markedly influences surface properties, particularly in ribbons annealed in $\mathrm{Ar}$ at $500^{\circ} \mathrm{C}$. Then, the surface action on the ribbon interior remains squeezing, but the compressive stress is significantly reduced by the substitution. This makes the loops less slant, more linear and reduces the coercivity although the magnetostriction is not reduced.

\section{Acknowledgments}

This work was partly financial supported by National Agency VEGA projects No. 2/0056/11, 2/0056/12 and $2 / 0111 / 11$. Authors are also grateful to Center of Excellence FUN-MAT.

\section{References}

[1] A. Urata, H. Matsumoto, S. Yoshida, A. Makino, IEEE Trans. Mag. 47, 3177 (2011).

[2] B. Butvinová, P. Butvin, E. Illeková, P. Švec Sr., G. Vlasák, D. Janičkovič in: Proc. Sci. Conf. Physics of Materials 2012 Eds. J. Tóthová, V. Lisý, FEEI, TU Košice 2012, p. 175.

[3] A. Hubert, R. Schäfer, Magnetic Domains, Springer, Berlin 1998.

[4] B. Butvinová. P. Butvin, M. Kuzminski, M. Kadlečíková, A. Ślawska-Waniewska, IEEE Trans. Mag. 48, 1340 (2012).

[5] G. Herzer, IEEE Trans. Mag. 30, 4800 (1994). 